\title{
Using Twitter to Surveil the Opioid Epidemic in North Carolina: An Exploratory Study
}

Mohd Anwar $^{1}$, PhD; Dalia Khoury ${ }^{2}$, PhD; Arnie P Aldridge ${ }^{2}$, PhD; Stephanie J Parker ${ }^{2}$, BA; Kevin P Conway ${ }^{2}$, PhD

${ }^{1}$ North Carolina A\&T State University, Greensboro, NC, United States

${ }^{2}$ Research Triangle Institute International, Research Triangle Park, NC, United States

Corresponding Author:

Mohd Anwar, PhD

North Carolina A\&T State University

1601 East Market St

Greensboro, NC, 27401

United States

Phone: 1 (336) 3347500

Email:

\section{Abstract}

Background: Over the last two decades, deaths associated with opioids have escalated in number and geographic spread, impacting more and more individuals, families, and communities. Reflecting on the shifting nature of the opioid overdose crisis, Dasgupta, Beletsky, and Ciccarone offer a triphasic framework to explain that opioid overdose deaths (OODs) shifted from prescription opioids for pain (beginning in 2000), to heroin (2010 to 2015), and then to synthetic opioids (beginning in 2013). Given the rapidly shifting nature of OODs, timelier surveillance data are critical to inform strategies that combat the opioid crisis. Using easily accessible and near real-time social media data to improve public health surveillance efforts related to the opioid crisis is a promising area of research.

Objective: This study explored the potential of using Twitter data to monitor the opioid epidemic. Specifically, this study investigated the extent to which the content of opioid-related tweets corresponds with the triphasic nature of the opioid crisis and correlates with OODs in North Carolina between 2009 and 2017.

Methods: Opioid-related Twitter posts were obtained using Crimson Hexagon, and were classified as relating to prescription opioids, heroin, and synthetic opioids using natural language processing. This process resulted in a corpus of 100,777 posts consisting of tweets, retweets, mentions, and replies. Using a random sample of 10,000 posts from the corpus, we identified opioid-related terms by analyzing word frequency for each year. OODs were obtained from the Multiple Cause of Death database from the Centers for Disease Control and Prevention Wide-ranging Online Data for Epidemiologic Research (CDC WONDER). Least squares regression and Granger tests compared patterns of opioid-related posts with OODs.

Results: The pattern of tweets related to prescription opioids, heroin, and synthetic opioids resembled the triphasic nature of OODs. For prescription opioids, tweet counts and OODs were statistically unrelated. Tweets mentioning heroin and synthetic opioids were significantly associated with heroin OODs and synthetic OODs in the same year $(P=.01$ and $P<.001$, respectively), as well as in the following year $(P=.03$ and $P=.01$, respectively). Moreover, heroin tweets in a given year predicted heroin deaths better than lagged heroin OODs alone $(P=.03)$.

Conclusions: Findings support using Twitter data as a timely indicator of opioid overdose mortality, especially for heroin.

(JMIR Public Health Surveill 2020;6(2):e17574) doi: 10.2196/17574

\section{KEYWORDS}

opioids; surveillance; social media

\section{Introduction}

Opioid overdose deaths (OODs) constitute a significant public health burden for the United States. In 2018, of the 67,367 drug overdose-related deaths, $70 \%(46,802)$ were attributed to opioids, with increases across demographic and geographic subgroups. Additionally, OODs involving synthetic opioids (eg, fentanyl) increased $10 \%$ from 2017 to 2018 and accounted for two-thirds of opioid-related deaths [1]. By contrast, rates of 
OODs involving heroin and prescription opioids decreased between 2017 and 2018 (by $4.1 \%$ and $13.5 \%$, respectively).

Reflecting on the evolving nature of the opioid crisis, Dasgupta, Beletsky, and Ciccarone [2] present an explanatory triphasic framework. The first phase, beginning in 2000, was based on prescription opioids for pain. The second involved a sharp increase in heroin overdose deaths between 2010 and 2015. The third phase saw a rapid increase in overdose deaths attributable to synthetic opioids, beginning in 2013.

Currently, the monitoring of OODs relies primarily on mortality data that lag between 12 to 18 months behind real time. Given the rapidly shifting nature of OODs, timelier surveillance data are critical to inform strategies that combat the opioid crisis. Over the last several years, there have been over 1000 health-related publications using Twitter to inform health research. This body of science spans a number of disparate areas, including tracking the spread of influenza [3,4], oral health problems [5], sleep issues [6], obesity [7], cardiovascular disease [8], diabetes [9], mental health [10], and health care enrollment [11]. In addition, there is burgeoning interest in the use of innovative and nontraditional methods (such as mining and analyzing social media data) as a means to better surveil the opioid epidemic, with Twitter becoming a complementary data source for pharmacovigilance $[12,13]$.

Regarding opioids specifically, researchers have analyzed Twitter messages and other social media posts from forums such as Reddit to understand their role in recovery from opioid use disorder [14], and access to and diversion of prescription drugs [15-18] and illicit opioids [19]. Twitter data have also been mined to study perceptions and attitudes toward opioids [20-22], including those held by specific groups such as youth [23]. Researchers have used other data streams, including Google Trends to forecast premature death from alcohol, drugs, and suicides [24]; a cryptomarket forum on the Dark Web to assess the emergence of new psychoactive substances [25]; and WebMD to explore motivations to use buprenorphine [26,27]. Recently, Graves et al [28] reported that thematic patterns of opioid-related tweets correlated with opioid overdose rates at the state and county levels. Sarker et al [29] reported that opioid-related tweets in Pennsylvania correlated with county-level OODs over 3 years. However, no study investigated whether opioid-related tweets in a given year can predict subsequent OODs.

This study explored Twitter data to monitor the opioid epidemic. Specifically, this study investigated the extent to which the content of opioid-related tweets corresponds with the triphasic nature of the opioid crisis and correlates with OODs in North Carolina between 2009 and 2017. North Carolina was selected because of its high rates of OODs, which increased notably during the study period.

\section{Methods}

Data collection from Twitter involved retrospectively monitoring the platform using Crimson Hexagon to access all English opioid-related posts from January 1, 2009, through December 31, 2018, in North Carolina. We created queries (opinion monitors) with a set of parameters (search terms) in Crimson Hexagon including commercial (eg, oxycodone, codeine, and morphine) and "street" names (eg, white, syrup, and tar) of drugs. We cast a broad net to capture terms referencing both trade and generic names. In order to identify such terms, we searched for common slang words referring to opioids using the Drug Enforcement Administration's (DEA) Intelligence Report titled "Slang Terms and Code Words: A Reference for Law Enforcement Personnel" [30]. We subsequently eliminated posts in which the slang term (eg, "China") appeared without any mention of the identified search term parameters elsewhere in that post. We excluded posts that contained hyperlinks as well as those containing solicitation-related words such as "buy" and "sell" as these were likely to be related to illegal online drug promotion or spamming techniques encouraging users to link to other sites.

Post location was determined through cross-verification of the geotag, profile information, time zones, content, and image data. This process resulted in a corpus of 100,777 posts consisting of tweets, retweets, mentions, and replies. We made the decision not to exclude retweets with the understanding that retweets signify a unique form of communication through an implied endorsement or agreement with the initial post [31].

Using a random sample of 10,000 posts from the corpus, we identified opioid-related terms by analyzing word frequency for each year. Next, we coded these terms into three tweet categories: prescription opioids (eg, codeine, morphine, pain, hydrocodone, pills, syrup, oxycodone, oxycontin, Percocet, and Vicodin), heroin (eg, heroin, tar, and white), and synthetic opioids (eg, fentanyl, synthetic, and laced).

We obtained annual mortality data from 2009 to 2018 from the Multiple Cause of Death database from the Centers for Disease Control and Prevention Wide-ranging Online Data for Epidemiologic Research (CDC WONDER) [32]. Drug overdose deaths were classified using the 10th revision of International Classification of Diseases (ICD-10), based on the ICD-10 underlying cause-of-death codes X40-X44 (unintentional), X60-X64 (suicide), X85 (homicide), or Y10-Y14 (undetermined intent). Drug overdoses with the following codes were considered OODs: opium (T40.0), heroin (T40.1), natural and semisynthetic opioids (T40.2), methadone (T40.3), synthetic opioids other than methadone (T40.4), and other unspecified narcotics (T40.6).

We estimated the association between the opioid-related tweet categories and OODs using ordinary least squares regression with either the current tweet count or a 1-year lag of tweet count as the independent variable. We also fit a vector autoregression and used Granger tests [33] to determine whether lagged tweet counts predict OODs better than lagged OODs alone. Stationarity for each of the six series was tested using an augmented Dickey-Fuller [34] unit root test with up to two lags and a linear trend. Analyses used Stata/MP (Version 15.1; StataCorp LLC).

This study consisted of secondary analyses; no individuals were involved. As data do not include any personally identifiable information, Institutional Review Board approval was not required. 


\section{Results}

The pattern of opioid-related Twitter posts in North Carolina appears in Figure 1A. Tweets about prescription opioids and heroin progressed in a similar, nonlinear pattern until they diverged in 2015, when heroin tweets increased and tweets for prescription opioids decreased. Tweets about synthetic opioids were virtually nonexistent until 2016, when they increased.
The progression of OODs in North Carolina appears in Figure 1B. Prescription opioids were the leading cause of OODs from 2009 to 2016. Heroin was the third leading cause of OODs until 2012, the second leading cause until 2016, and the third leading cause in 2017. Fentanyl became the leading cause of OODs in 2017.

Figure 1. Pattern of opioid-related deaths and tweets in North Carolina over time.
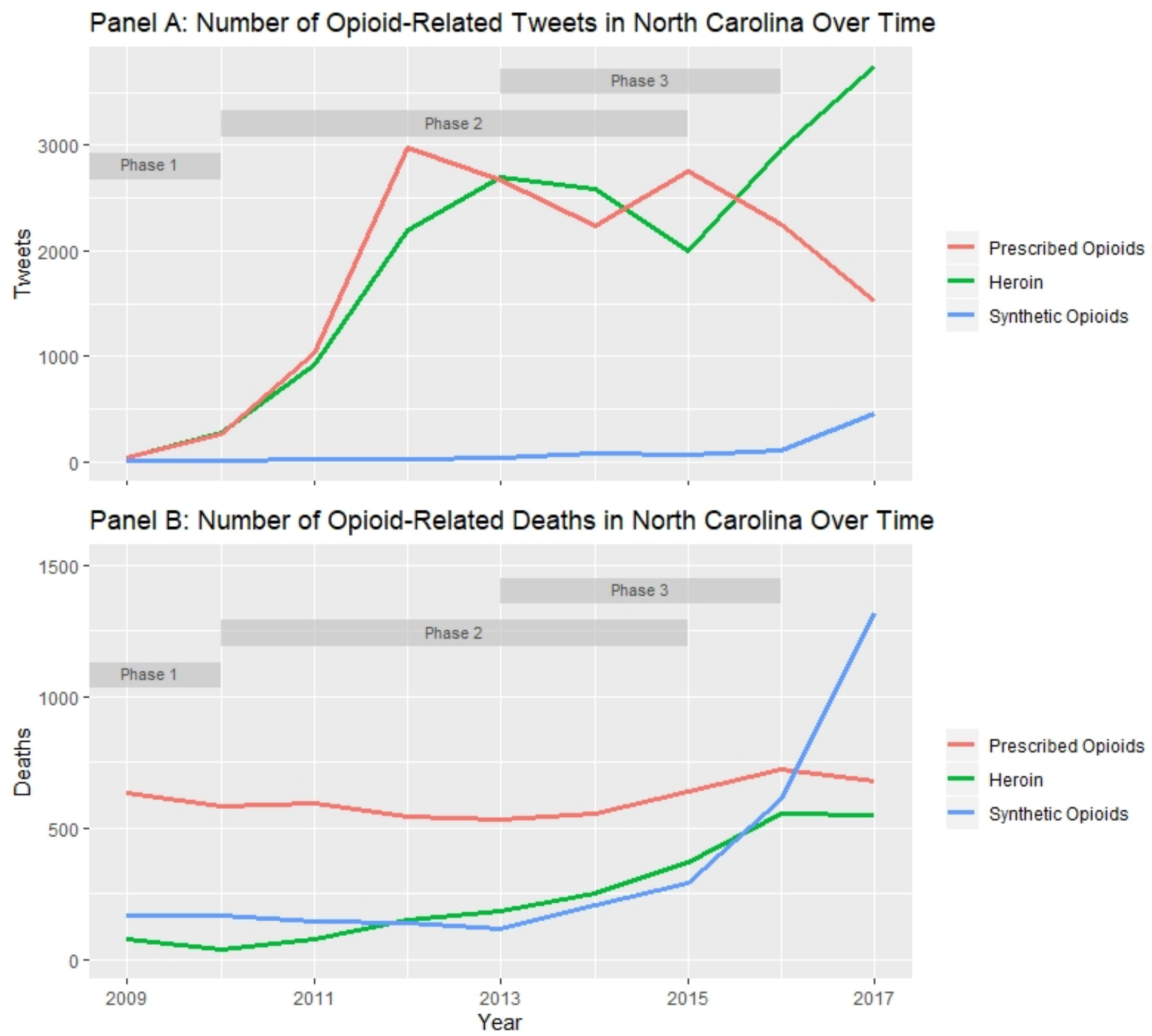

Using the augmented Dickey-Fuller tests, we failed to reject stationarity up to two lags for all variables except for prescription OODs. The association between tweet count and OODs was not significant for prescription opioids in either the one-year lag model (coefficient $=0.01 ; P=.58$ ) or the no-lag model (coefficient $=0.01 ; P=.64)$. In contrast, tweet counts for both heroin and synthetic opioids were significantly associated with OODs. On average, each additional heroin tweet in a given year corresponded to 0.13 additional heroin overdose deaths that same year $(P=.01)$ and 0.13 additional deaths the following year $(P=.03)$. Each additional tweet mentioning synthetic opioids in a given year corresponded to 2.68 additional synthetic opioid overdose deaths that year $(P<.001)$ and 9.24 additional deaths the next year $(P=.01)$.

Granger tests following vector autoregression estimation with one and two lags (only one lag was estimated for prescription OODs) were consistent with the regression results but significant only for heroin tweets; tweets mentioning heroin in a given year significantly predicted subsequent heroin OODs $(P=.03)$ over and above lagged heroin OODs. 


\section{Discussion}

The pattern of opioid-related Twitter posts in North Carolina resembled the triphasic nature of the opioid crisis as described by Dasgupta et al [2]. Tweets about prescription opioids and heroin were intertwined through the end of Phase 2, when tweets about prescription opioids declined and tweets about heroin surged. During Phase 3, tweets about synthetic opioids emerged around 2016.

Results from the regression models and Granger tests indicated that the association with OODs differed by the type of opioid. For prescription opioids, tweet count and OODs were unrelated. The lack of association observed between prescription opioid tweets and overdose deaths may underestimate the true association therein, particularly because some patients who are treated with prescription opioids are chronic pain patients or older individuals [35], who may be less likely to have an active presence on Twitter. Indeed, almost half of Twitter users are aged 18 to 24 years (44\%), followed by those aged 25 to 29 years (31\%), 30 to 49 years (26\%), 50 to 64 years (17\%), and $\geq 65$ years (7\%) [36]. Although our sample may underestimate the association among individuals aged 50 years and older, this bias seems likely to be minimal because the majority of opioid overdose-related deaths in 2018 occurred among individuals aged 25 to 44 years [1].

Tweets mentioning heroin and synthetic opioids were significantly associated with heroin OODs and synthetic OODs, respectively. Moreover, results from the Granger tests showed that heroin tweets in a given year predicted subsequent heroin deaths better than lagged heroin OODs alone. These predictive results extend recent reports of correlations between opioid-related tweets and opioid overdose rates at the state and county levels $[28,29]$.

There are a number of limitations to be considered. First, the scope of the terms used in our search parameters was somewhat subjective, in that there are hundreds of terms representing opioids [30], and we selected the most frequently used terms.
This may have underestimated the breadth of opioid-related tweets in our sample. Second, we were limited in our ability to validate whether a tweet was indeed about opioids, as it was not possible to identify and query the tweet author about his or her intention. However, research on social media discussions related to cardiovascular mortality [37] and depression [38,39] indicate that these discussions reflect behavioral intentions. Third, filtering out solicitation-related terms and posts with hyperlinks was predicated on the assumption that these tweets reflect illicit opioid sales, which may constitute a unique phenomenon. Indeed, Katsuki et al [15] found that $75.2 \%$ of tweets containing URLs linked to an illicit online pharmacy, and Mackey et al [16] found that $90 \%$ of online marketing tweets included hyperlinks. Our decision certainly reduced the number of posts in our sample and may have resulted in misclassification. However, given that the overwhelming majority of individuals who misuse opioids report obtaining opioids from friends and family [40], it is likely that this decision had only a small impact on our results. Future research should examine whether tweets that include drug solicitation terms correlate with overdose rates in ways that differ from posts that exclude such terms. Finally, we were limited to correlational analyses without statistical controls, due to insufficient time points needed to run more sophisticated analyses. Our results should be considered preliminary; more research is needed with additional time points and data before making definitive statements.

Limitations notwithstanding, to our knowledge, this study is the first to report that the pattern of opioid-related Twitter posts in North Carolina not only resembles the triphasic nature of the opioid crisis [2], but that tweets mentioning heroin and synthetic opioids also correlate with and predict OODs. Findings suggest that Twitter data should be further evaluated as a novel and timely indicator of opioid overdose mortality, especially for heroin. Twitter use is widespread; of the 68 million Twitter users in the United States, $87 \%$ keep their feed public, nearly half of whom report daily usage [41]. Thus, tweets have the potential to serve as a readily available, unique, and real-time data source for surveilling the opioid crisis.

\section{Acknowledgments}

This research was supported by funding from the Research Triangle Institute (RTI) Opioid Strategic Investment Fund. The funder had no role in the design and conduct of the study; collection, management, analysis, and interpretation of the data; preparation, review, or approval of the manuscript; and decision to submit the manuscript for publication.

\section{Authors' Contributions}

MA, DK, and KPC contributed to the study concept and design. All authors contributed to the acquisition, analysis, or interpretation of data. APA did the statistical analysis. DK, KPC, MA, and APA drafted the manuscript. All authors critically revised the manuscript for important intellectual content. DK and SJP provided administrative, technical, or material support. KPC obtained funding and provided supervision.

\section{Conflicts of Interest}

None declared.

\section{References}


1. Wilson N, Kariisa M, Seth P, Smith H, Davis NL. Drug and Opioid-Involved Overdose Deaths - United States, $2017-2018$. MMWR Morb Mortal Wkly Rep 2020 Mar 20;69(11):290-297 [FREE Full text] [doi: 10.15585/mmwr.mm6911a4] [Medline: 32191688]

2. Dasgupta N, Beletsky L, Ciccarone D. Opioid Crisis: No Easy Fix to Its Social and Economic Determinants. Am J Public Health 2018 Feb;108(2):182-186. [doi: 10.2105/ajph.2017.304187] [Medline: 29267060]

3. Woo H, Sung Cho H, Shim E, Lee JK, Lee K, Song G, et al. Identification of Keywords From Twitter and Web Blog Posts to Detect Influenza Epidemics in Korea. Disaster Med Public Health Prep 2017 Jul 31;12(3):352-359. [doi: 10.1017/dmp.2017.84] [Medline: 28756796]

4. Paul MJ, Dredze M, Broniatowski D. Twitter Improves Influenza Forecasting. PLoS Curr 2014. [doi: 10.1371/currents.outbreaks.90b9ed0f59bae4ccaa683a39865d9117] [Medline: 25642377]

5. Heaivilin N, Gerbert B, Page J, Gibbs J. Public Health Surveillance of Dental Pain via Twitter. J Dent Res 2011 Jul 18;90(9):1047-1051. [doi: 10.1177/0022034511415273] [Medline: 21768306]

6. McIver DJ, Hawkins JB, Chunara R, Chatterjee AK, Bhandari A, Fitzgerald TP, et al. Characterizing Sleep Issues Using Twitter. J Med Internet Res 2015 Jun 08;17(6):e140 [FREE Full text] [doi: 10.2196/jmir.4476] [Medline: 26054530]

7. Anwar M, Yuan Z. Linking Obesity Tweets. In: Zheng X, Zeng D, Chen H, Leischow S, editors. ICSH 2015. Lecture Notes in Computer Science, vol. 9545. Cham: Springer; 2016.

8. Sinnenberg L, DiSilvestro CL, Mancheno C, Dailey K, Tufts C, Buttenheim AM, et al. Twitter as a Potential Data Source for Cardiovascular Disease Research. JAMA Cardiol 2016 Dec 01;1(9):1032. [doi: 10.1001/jamacardio.2016.3029] [Medline: 27680322]

9. Griffis H, Asch DA, Schwartz HA, Ungar L, Buttenheim AM, Barg FK, et al. Using Social Media to Track Geographic Variability in Language About Diabetes: Analysis of Diabetes-Related Tweets Across the United States. JMIR Diabetes 2020 Jan 26;5(1):e14431 [FREE Full text] [doi: 10.2196/14431] [Medline: 32044757]

10. Guntuku SC, Yaden DB, Kern ML, Ungar LH, Eichstaedt JC. Detecting depression and mental illness on social media: an integrative review. Current Opinion in Behavioral Sciences 2017 Dec;18:43-49. [doi: 10.1016/j.cobeha.2017.07.005]

11. Wong CA, Sap M, Schwartz A, Town R, Baker T, Ungar L, et al. Twitter sentiment predicts Affordable Care Act marketplace enrollment. J Med Internet Res 2015 Feb 23;17(2):e51 [FREE Full text] [doi: 10.2196/jmir.3812] [Medline: 25707038]

12. Brownstein JS, Freifeld CC, Madoff LC. Digital Disease Detection - Harnessing the Web for Public Health Surveillance. N Engl J Med 2009 May 21;360(21):2153-2157. [doi: 10.1056/nejmp0900702] [Medline: 19423867]

13. Lardon J, Bellet F, Aboukhamis R, Asfari H, Souvignet J, Jaulent M, et al. Evaluating Twitter as a complementary data source for pharmacovigilance. Expert Opinion on Drug Safety 2018 Jul 26;17(8):763-774. [doi: 10.1080/14740338.2018.1499724] [Medline: 29991282]

14. D'Agostino AR, Optican AR, Sowles SJ, Krauss MJ, Escobar Lee K, Cavazos-Rehg PA. Social networking online to recover from opioid use disorder: A study of community interactions. Drug and Alcohol Dependence 2017 Dec;181:5-10. [doi: 10.1016/j.drugalcdep.2017.09.010] [Medline: 29024875]

15. Katsuki T, Mackey TK, Cuomo R. Establishing a Link Between Prescription Drug Abuse and Illicit Online Pharmacies: Analysis of Twitter Data. J Med Internet Res 2015 Dec 16;17(12):e280 [FREE Full text] [doi: 10.2196/jmir.5144] [Medline: 26677966]

16. Mackey TK, Kalyanam J, Katsuki T, Lanckriet G. Twitter-Based Detection of Illegal Online Sale of Prescription Opioid. Am J Public Health 2017 Dec;107(12):1910-1915. [doi: 10.2105/ajph.2017.303994] [Medline: 29048960]

17. Kalyanam J, Mackey TK. A Review of Digital Surveillance Methods and Approaches to Combat Prescription Drug Abuse. Curr Addict Rep 2017 Sep 18;4(4):397-409. [doi: 10.1007/s40429-017-0169-4]

18. Chary M, Genes N, Giraud-Carrier C, Hanson C, Nelson LS, Manini AF. Epidemiology from Tweets: Estimating Misuse of Prescription Opioids in the USA from Social Media. J Med Toxicol 2017 Dec;13(4):278-286 [FREE Full text] [doi: 10.1007/s13181-017-0625-5] [Medline: 28831738]

19. Mackey T, Kalyanam J, Klugman J, Kuzmenko E, Gupta R. Solution to Detect, Classify, and Report Illicit Online Marketing and Sales of Controlled Substances via Twitter: Using Machine Learning and Web Forensics to Combat Digital Opioid Access. J Med Internet Res 2018 Apr 27;20(4):e10029 [FREE Full text] [doi: 10.2196/10029] [Medline: 29613851]

20. Shutler L, Perrone J, Portelli I, Nelson L, Blachford C. Prescription Opioids in the Twittersphere: A Contextual Analysis of Tweets About Prescription Drugs. Annals of Emergency Medicine 2013 Oct;62(4):S122. [doi: 10.1016/j.annemergmed.2013.07.169] [Medline: 26364675]

21. Glowacki EM, Glowacki JB, Wilcox GB. A text-mining analysis of the public's reactions to the opioid crisis. Substance Abuse 2017 Sep 20;39(2):129-133. [doi: 10.1080/08897077.2017.1356795] [Medline: 28723265]

22. Chan B, Lopez A, Sarkar U. The Canary in the Coal Mine Tweets: Social Media Reveals Public Perceptions of Non-Medical Use of Opioids. PLoS ONE 2015 Aug 7;10(8):e0135072. [doi: 10.1371/journal.pone.0135072] [Medline: 26252774]

23. Stevens RC, Brawner BM, Kranzler E, Giorgi S, Lazarus E, Abera M, et al. Exploring Substance Use Tweets of Youth in the United States: Mixed Methods Study. JMIR Public Health Surveill 2020 Mar 26;6(1):e16191 [FREE Full text] [doi: 10.2196/16191] [Medline: $\underline{32213472]}$ 
24. Parker J, Cuthbertson C, Loveridge S, Skidmore M, Dyar W. Forecasting state-level premature deaths from alcohol, drugs, and suicides using Google Trends data. Journal of Affective Disorders 2017 Apr;213:9-15. [doi: 10.1016/j.jad.2016.10.038] [Medline: 28171770 ]

25. Van Hout MC, Hearne E. New psychoactive substances (NPS) on cryptomarket fora: An exploratory study of characteristics of forum activity between NPS buyers and vendors. International Journal of Drug Policy 2017 Feb;40:102-110. [doi: 10.1016/j.drugpo.2016.11.007] [Medline: 28027812]

26. Butler SF, Oyedele NK, Dailey Govoni T, Green JL. How Motivations for Using Buprenorphine Products Differ From Using Opioid Analgesics: Evidence from an Observational Study of Internet Discussions Among Recreational Users. JMIR Public Health Surveill 2020 Mar 25;6(1):e16038 [FREE Full text] [doi: 10.2196/16038] [Medline: 32209533]

27. Rivas R, Sadah S, Guo Y, Hristidis V. Classification of Health-Related Social Media Posts: Evaluation of Post Content-Classifier Models and Analysis of User Demographics. JMIR Public Health Surveill 2020 Apr 01;6(2):e14952 [FREE Full text] [doi: 10.2196/14952] [Medline: $\underline{32234706}$ ]

28. Graves RL, Tufts C, Meisel ZF, Polsky D, Ungar L, Merchant RM. Opioid Discussion in the Twittersphere. Substance Use \& Misuse 2018 Apr 16;53(13):2132-2139. [doi: 10.1080/10826084.2018.1458319] [Medline: 29659320]

29. Sarker A, Gonzalez-Hernandez G, Ruan Y, Perrone J. Machine Learning and Natural Language Processing for Geolocation-Centric Monitoring and Characterization of Opioid-Related Social Media Chatter. JAMA Netw Open 2019 Nov 06;2(11):e1914672. [doi: 10.1001/jamanetworkopen.2019.14672] [Medline: 31693125]

30. Drug Enforcement Administration. Drug Enforcement Administration. In: Drug Enforcement Administration: A tradition of excellence 1973-2008. Washington DC: US Department of Justice; 2008.

31. Boyd D, Golder S, Lotan G. Tweet, tweet, retweet: Conversational aspects of retweeting on Twitter. In: HICSS-43. IEEE. 2010 Presented at: HICSS-43 - IEEE; January 2010; Kauai, HI p. 6. [doi: 10.1109/hicss.2010.412]

32. Centers for Disease Control and Prevention, National Center for Health Statistics. Multiple Cause of Death Files $1999-2017$ on CDC WONDER Online Database. 2018 Dec. Multiple Cause of Death 1999-2017 on CDC WONDER Online Database URL: http://wonder.cdc.gov/mcd-icd10.html [accessed 2019-06-28]

33. Granger CWJ. Investigating Causal Relations by Econometric Models and Cross-spectral Methods. Econometrica 1969 Aug;37(3):424. [doi: 10.2307/1912791]

34. Dickey DA, Fuller WA. Distribution of the Estimators for Autoregressive Time Series with a Unit Root. Journal of the American Statistical Association 1979 Jun;74(366a):427-431. [doi: 10.1080/01621459.1979.10482531]

35. Olfson M, Wang S, Iza M, Crystal S, Blanco C. National trends in the office-based prescription of schedule II opioids. J Clin Psychiatry 2013 Sep;74(9):932-939. [doi: 10.4088/JCP.13m08349] [Medline: 24107767]

36. Perrin A, Andersen M. Pew Research. 2019 Apr 10. Share of U.S. adults using social media, including Facebook, is mostly unchanged since 2018 URL: https://www.pewresearch.org/fact-tank/2019/04/10/

share-of-u-s-adults-using-social-media-including-facebook-is-mostly-unchanged-since-2018/ [accessed 2019-07-05]

37. Eichstaedt JC, Schwartz HA, Kern ML, Park G, Labarthe DR, Merchant RM, et al. Psychological language on Twitter predicts county-level heart disease mortality. Psychol Sci 2015 Jan 20;26(2):159-169. [doi: 10.1177/0956797614557867] [Medline: 25605707]

38. Nambisan P, Luo Z, Kapoor A, Patrick T, Cisler R. Social media, big data, and public health informatics: Ruminating behavior of depression revealed through twitter. 2015 Presented at: 48th International Conference on System Sciences (HICSS); 2015 Jan 5; Hawaii p. 2906-2913. [doi: 10.1109/hicss.2015.351]

39. Mowery D, Smith HA, Cheney T, Bryan C, Conway M. Identifying Depression-Related Tweets from Twitter for Public Health Monitoring. OJPHI 2016 Mar 24;8(1). [doi: 10.5210/ojphi.v8i1.6561]

40. US Department of Health and Human Services, Substance Abuse and Mental Health Services Administration, Center for Behavioral Health Statistics and Quality. National Survey on Drug Use and Health 2018 (NSDUH-2018-DS0001). 2018. URL: https://datafiles.samhsa.gov/ [accessed 2019-07-02]

41. Remy E. Medium. 2015 Jul 15. How public and private Twitter users in the U.S. compare URL: https://medium.com/ pew-research-center-decoded/how-public-and-private-twitter-users-in-the-u-s-d536ce2a41b3 [accessed 2019-07-30]

\section{Abbreviations}

CDC: Centers for Disease Control and Prevention

DEA: Drug Enforcement Administration

ICD-10: International Classification of Diseases, 10th revision

OOD: opioid overdose death

WONDER: Wide-ranging Online Data for Epidemiologic Research 
Edited by T Sanchez; submitted 31.12.19; peer-reviewed by D Broniatowski, A Kulanthaivel, C Freiermuth; comments to author 03.03.20; revised version received 27.04.20; accepted 15.05.20; published 24.06.20

Please cite as:

Anwar M, Khoury D, Aldridge AP, Parker SJ, Conway KP

Using Twitter to Surveil the Opioid Epidemic in North Carolina: An Exploratory Study

JMIR Public Health Surveill 2020;6(2):e17574

URL: http://publichealth.jmir.org/2020/2/e17574/

doi: $10.2196 / 17574$

PMID: $\underline{32469322}$

CMohd Anwar, Dalia Khoury, Arnie P Aldridge, Stephanie J Parker, Kevin P Conway. Originally published in JMIR Public Health and Surveillance (http://publichealth.jmir.org), 24.06.2020. This is an open-access article distributed under the terms of the Creative Commons Attribution License (https://creativecommons.org/licenses/by/4.0/), which permits unrestricted use, distribution, and reproduction in any medium, provided the original work, first published in JMIR Public Health and Surveillance, is properly cited. The complete bibliographic information, a link to the original publication on http://publichealth.jmir.org, as well as this copyright and license information must be included. 